\title{
Hautkrebs: Jedes Jahr fünf Prozent mehr Neuerkrankungen
}

Hautkrebs hat sich in den vergangenen Jahrzehnten stark ausgebreitet, in Europa steigt die Zahl der Neuerkrankungen jährlich um fünf Prozent. In Österreich erkranken pro Jahr 1500 Menschen an einem malignen Melanom und geschätzte 30.000 an Weißem Hautkrebs. Heute kann Hautkrebs frühzeitig entdeckt und durch eine frühe Therapie auch in den meisten Fällen geheilt werden. Allerdings sind die neuen Therapien nicht für alle Patienten in Europa zugänglich.

Quelle: Presseaussendung B\&K zum 16th World Congress on Cancers of the Skin und dem 12th Congress of the European Association of Dermato-Oncology, 31. August bis 3. September 2016, Wien

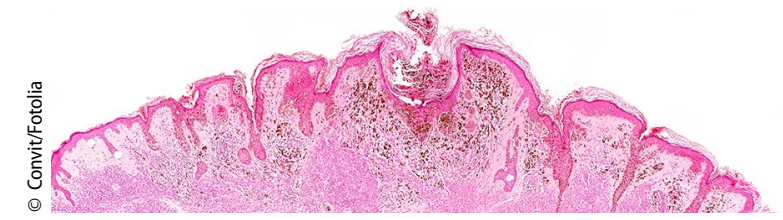

Abb. 1 \ Nach dem Zweiten Weltkrieg lag die Inzidenz des Melanoms bei einem Fall pro 100.000 Einwohner und Jahr. Inzwischen rangiert es in den meisten westeuropäischen Ländern an den Plätzen 4 bis 7 der häufigsten Krebsarten te EADO-Präsident Prof. Dr. Claus Garbe von der Universitäts-Hautklinik Tübingen. „Nach dem Zweiten Weltkrieg lag die Inzidenz des Melanoms bei einem Fall pro 100.000 Einwohner und Jahr. Inzwischen rangiert es in den meisten westeuropäischen Ländern an den Plätzen 4 bis 7 der häufigsten Krebsarten."

Gegenwärtig sind in den nordund westeuropäischen Ländern pro Jahr zwischen 15 und 25 Personen pro 100.000 Einwohner vom "schwarzen Hautkrebs" betroffen. Auch die Fälle von Hautkrebsformen ohne Melanom, etwa das Basalzellkarzinom und Plattenepithelkarzinom, sind in den letzten vier Jahrzehnten enorm angestiegen. Garbe: „Prognosen erwarten für die nächsten 15 Jahre zumindest eine Verdoppelung der Hautkrebs-Inzidenz-Raten."

Dafür gibt es einen weithin bekannten Grund: Sonnenbaden ist durch die veränderten Urlaubs- und Freizeitgewohnheiten seit der Mitte des 20. Jahrhunderts weit verbreitet, und allen Hautkrebsformen ist gemeinsam, dass das UV-Licht der Sonne einer der auslösenden Faktoren ist.

\section{Europäische Drei-Klassen-Medizin}

Alle Hautkrebsarten können heute bei entsprechender Kontrolle durch den Arzt prinzipiell frühzeitig entdeckt und durch eine frühe Therapie auch in den allermeisten Fällen geheilt werden. In den letzten zehn Jahren wurde eine Reihe vielversprechender Therapien gegen Hautkrebs entwickelt. Wie eine EADOStudie zeigt, sind die neuen Therapien allerdings nicht für alle Patienten in den eu- ropäischen Staaten zugänglich, so Garbe: „Vor allem in Ost- und Südosteuropa gibt es große Versorgungsdefizite: Rund 5000 Patientinnen und Patienten pro Jahr würden von den neuen Medikamenten profitieren, doch sie sind unerschwinglich, weder für Gesundheitssysteme noch für Privatpersonen leistbar." [1]

„De facto gibt es in Europa eine DreiKlassen-Medizin, was den Zugang zu neuen Hautkrebs-Medikamenten betrifft", erklärte Garbe: In Ländern wie Deutschland ist mit der Zulassung eines Medikamentes auch dessen Finanzierung durch die Krankenkassen gesichert und das Mittel steht somit den Patienten zur Verfügung. In der Mehrzahl der westeuropäischen Länder müssen zunächst Preisverhandlungen stattfinden, die sich auch über mehrere Jahre hinziehen können. Erst danach werden die Kosten für die Medikamente erstattet, wobei es hier Einschränkungen in einzelnen Staaten geben kann. „Am schwersten ist der Zugang zu den neuen Therapien für die Betroffenen in den meisten osteuropäischen Staaten einschließlich Russland“, so Garbe. „Diese Länder können es sich nicht leisten, die Kosten für die Medikamente zu tragen.“

\section{Literatur \\ 1. Sekulovic et al (2016) More than 5000 pa- tients with metastatic melanoma in Europe per year do not have access to the new life-saving drugs. ESMO Abstract 4588}

hautnah $2016 \cdot 15: 100$

DOI 10.1007/s12326-016-0219-z

Online publiziert: 12. Oktober 2016

(c) Springer-Verlag Wien 2016 